\title{
Standardization of Protocol for the Preparation of Linseed Beverage Using Lactic Acid Bacteria and Yeast
}

\author{
N. Nivetha ${ }^{1} *$, V.C. Suvarna ${ }^{2}$ and A.J. Shraddha ${ }^{2}$ \\ ${ }^{1}$ University of Agricultural Sciences, GKVK, Bangalore, Karnataka, India-560065 \\ ${ }^{2}$ Department of Microbiology, UAS, GKVK Bangalore, Karnataka, India-560065 \\ *Corresponding author
}

\section{A B S T R A C T}

\section{Keywords}

Linseed, Lactic acid bacteria,

Saccharomyces,

Fermentation.

Article Info

Accepted:

22 February 2017

Available Online:

10 March 2017
Linseed is one of the most important crops and it has exceptionally high content of AlphaLinolenic acid, dietary fiber, high quality protein, phytoestrogens and minerals especially phosphorus, magnesium and calcium. To increase the uptake of linseed, value addition is attempted. Standardization of protocol was carried out based on $\mathrm{pH}$, titrable acidity and sensory characteristics for the parameters - seed processing technique, substrate, sugar, inoculum, honey concentrations, incubation temperature and fermentation duration using Lactobacillus acidophilus and Saccharomyces ellipsoideus. Seed processing technique of roasting and powdering, substrate concentration of $10 \%$ and $5 \%$, sugar concentration of $6.6 \%$ and $10 \%$ with sugar and honey in the ratio of 80:20 and 60:40 were selected for bacterial and yeast fermentation respectively. Incubation temperature of $30^{\circ} \mathrm{C}$ for 2 days with $8 \%$ inoculum was found to be effective for the preparation of fermented beverage. Based on the experimental results standard protocol was constructed and fermented beverage is prepared using probiotic organisms Lactobacillus acidophilus, Bacillus mesentericus, Saccharomyces ellipsoideus, Saccharomyces boulardii and lactic acid bacterial isolate. Organoleptic evaluation recorded an overall acceptability of 6.7 to 7.5. Cost of the beverage ranged from rupees 66.05 - 33.16 due to the variations in amount of recovery.

\section{Introduction}

Linseed also known as "flax" is one of the oldest cultivated crops grown by man for food and fiber. Linseed, Linum usitatissimum L. belongs to family Linaceae. It is one of the most important industrial oilseed crops of the world and is cultivated over an area of 3.02 million hectares with a production of 2.57 million tonnes and productivity of $852 \mathrm{~kg} / \mathrm{ha}$. India ranks first in terms of area under linseed cultivation with 4.68 lakh ha and third in production with 1.68 lakh tonnes and $349 \mathrm{~kg}$ /ha productivity.
Linseed is rich in lipids, protein and dietary fiber. Linseed contains 41, 20, 28, 7.7 and 3.4 per cent of fat, protein, dietary fiber, moisture and ash respectively (Morris, 2003). It is the richest known vegetable source of AlfaLinolenic acid (ALA) which is an omega-3fatty acid, phytoestrogen lignans and dietary fibers (Giada, 2010). Alfa-Linolenic acid is beneficial for cardiovascular diseases and it is known to reduce blood lipids. Linseed contains high quality protein with amino acids like arginine, aspartic acid and glutamic acid. 
Dietary fibers help to reduce constipation, to keep better bowel movement and as a hypo cholesterolemic agent. Lignans act as antioxidants, thereby decreasing the presence of free oxygen radical and it is also having anticancer property. These properties make it more favourable for food technologists to explore and develop nutraceutical foods.

Salminen et al., (2007) formulated a fermented beverage from linseed. Defatted and crushed linseed was mixed with other cereals, supplements, fermented with probiotic organisms like Lactobacillus and Bifidobacterium, stabilized using pectin or gum and seasoned with fruits like berries to develop a beverage. Ground linseed based refreshments accompanied by a little lemon juice is commonly observed in Bolivia, Peru and Colombia. Raw or roasted linseed was mixed with water, a few cloves, cinnamon and nutmeg and boiled for $15 \mathrm{~min}$. Then it is added with lemon juice and served after refrigeration. Because of its nutritional properties this is used as an energy drink in Bolivia.

Presence of Alpha-Linolenic acid, dietary fiber and lignans especially Secoisolariciresinol diglucoside (SDG) in linseed attract food technologists and exploration of its abilities is needed to be used in food processing sector. In this study an attempt was made to prepare a value added product from linseed by fermentation. Standardization of methodology was studied using Lactobacillus acidophilus and Saccharomyces ellipsoideus to open a way for large scale production.

\section{Materials and Methods}

Standardization of protocol to develop fermented product form linseed was studied. Lactobacillus acidophilus (MTCC-10307) obtained from MTCC, Chandigarh and Saccharomyces ellipsoideus $\left(\mathrm{SY}_{2}\right)$ (NCIM-
3200) from NCIM, Pune were used as reference cultures. Observations like $\mathrm{pH}$ (Digital pH meter type MK-VI), titrable acidity (Srivastava and Kumar, 1993) and sensory evaluation were considered for standardization.

Inoculum preparation method is given in figure 1. Population was determined by standard plate count method on MRS medium for lactic acid bacteria and Sabourd's agar medium for yeast $\mathrm{SY}_{2}$ (Salminen et al., 2008). Fermentation medium was prepared using required concentration of linseed, sugar, honey and inoculum. Samples were incubated at room temperature for fermentation until the standardization of temperature. Standardized parameters were considered for further experiments to standardize other parameters. Observations were taken at $2^{\text {nd }}, 3^{\text {rd }}$ and $4^{\text {th }}$ day of fermentation.

Parameters studied were as follows

1. Seed treatment - raw seed or roasted seed; inoculated or unioculated

2. Substrate concentration $-5,10$ and $15 \%$

3. Sugar concentration $-5,6.6,8$ and $10 \%$

4. Inoculum concentration $-6,8$ and $10 \%$.

5. Honey concentration - sugar and honey in 100:0, 80:20, 60:40 and 50:50.

6. Incubation temperature- 28,30 and $32^{\circ} \mathrm{C}$.

7. Fermentation duration $-2,3$ and 4 days.

Based on the experimental results standard protocol was constructed. Linseed fermented beverages using probiotic organisms Lactobacillus acidophilus, Bacillus mesentericus, Saccharomyces ellipsoideus, Saccharomyces boulardii and lactic acid bacterial isolate of linseed were prepared. Sensory evaluation was carried out for fermented linseed beverage by following 9 point hedonic scale by a panel of 17 judges. Cost and recovery of beverage was also calculated. 


\section{Results and Discussion}

The protocol was developed for preparing fermented beverage of linseed considering parameters like seed processing; incubation temperature and duration; effective isolate selection and concentration of substrate, sugar, inoculum and honey. Biochemical characteristics including $\mathrm{pH}$, titrable acidity and sensory characteristics like foam, flavor and taste were also considered for standardization.

Standardization for seed processing (Table 2) was tested and treatment $\left(\mathrm{T}_{8}\right)$ roasted, powdered with inoculation was selected for fermentation studies. This may be due to less amount of mucin compounds (form slimy sediment), easy availability of nutrients to microorganisms by powdering. Roasting process eliminates natural microbiota associated with linseed and there will be no competition for inoculum. Hence, in roasted treatment, the desired characteristics contributed by inoculum improve the quality of beverage.

Substrate concentrations (Table 3, 3A, 4 and 4A) of 10 per cent with 6.6 percent sugar concentration for lactic acid bacterial fermentation and 5 percent substrate with 10 per cent sugar concentration for yeast fermentation were preferred for beverage preparation. Biochemical characteristics like $\mathrm{pH}$ and titrable acidity in substrate concentrations 10 per cent and 15 per cent in lactic acid bacterial fermentation were on par with each other, thus 10 per cent concentration was selected for further studies.
Substrate concentration of 5 per cent with 10 per cent sugar concentration yielded good results for fermentation of linseed using yeasts. This could be due to the availability of balanced concentration of carbohydrates (sugars) and substrate for fermentation by yeast.

Inoculum of 36-48 hrs old culture was used for fermentation. Population of inoculum was found to decrease after 48 hrs incubation (Table 1). This could be attributed to the fact that cultures might have entered stationary and death phase after $48 \mathrm{hrs}$ incubation. Different inoculum levels (Table 5 and 5A) were added to substrate and results revealed that $\mathrm{pH}$ and titrable acidity increased with increase in inoculum levels. Inoculum concentration of 8 per cent and 10 per cent showed significant improvement in beverage properties, thus 8 per cent concentration was selected for fermented beverage preparation. Similar results were reported by Panesar et al., (2010), for lactic acid production.

Drastic reduction in $\mathrm{pH}$ and more titrable acid production was observed by the supplementation of honey with fermentation medium. Similar results were earlier reported by Macedoi et al., (2008), in 12 per cent nonfat dry milk containing 3 per cent of pasteurized honey fermented with Lactobacillus spp. and Bifidobacterium spp. Sugar and honey in the ratio of 8:2 and 6.5:3.5 for lactic acid bacterial and yeast fermentations were selected due to its favorable biochemical and sensory characteristics (Table 6 and 6A).

Table.1 Population density of inocula (cfu/ml)

\begin{tabular}{|l|l|l|}
\hline Inoculum & $\mathbf{2}^{\text {nd }}$ day & $\mathbf{3}^{\text {rd }}$ day \\
\hline Lactobacillus acidophilus & $4.63 \times 10^{7} \mathrm{cfu} / \mathrm{ml}$ & $1.46 \times 10^{7} \mathrm{cfu} / \mathrm{ml}$ \\
\hline Saccharomyces ellipsoideus $\left(\mathrm{SY}_{2}\right)$ & $16.1 \times 10^{5} \mathrm{cfu} / \mathrm{ml}$ & $6.9 \times 10^{5} \mathrm{cfu} / \mathrm{ml}$ \\
\hline
\end{tabular}


Table.2 Standardization for seed processing for fermented beverage production

\begin{tabular}{|l|c|c|l|l|c|}
\hline \multicolumn{1}{|c|}{ Treatments } & pH & TA & \multicolumn{1}{c|}{ Froth } & \multicolumn{1}{c|}{ Flavour } & Taste \\
\hline $\mathrm{T}_{1}-$ Raw seed + un inoculated & $5.54^{\mathrm{e}}$ & $0.05^{\mathrm{bc}}$ & More & Bad & - \\
\hline $\mathrm{T}_{2}-$ Raw seed + inoculated & $5.31^{\mathrm{d}}$ & $0.05^{\mathrm{bc}}$ & More & Bad & - \\
\hline $\begin{array}{l}\mathrm{T}_{3}-\text { Raw seed, powdered }+ \\
\text { un inoculated }\end{array}$ & $4.73^{\mathrm{b}}$ & $0.42^{\mathrm{a}}$ & Very less & Bad & - \\
\hline $\begin{array}{l}\mathrm{T}_{4}-\text { Raw seed, powdered + } \\
\text { inoculated }\end{array}$ & $4.34^{\mathrm{a}}$ & $0.41^{\mathrm{a}}$ & Very less & Not good & More sour taste \\
\hline $\begin{array}{l}\mathrm{T}_{5}-\text { Roasted seed + un } \\
\text { inoculated }\end{array}$ & $5.28^{\mathrm{d}}$ & $0.04^{\mathrm{c}}$ & Very less & Not bad & Less sour taste \\
\hline $\begin{array}{l}\mathrm{T}_{6}-\text { Roasted seed + } \\
\text { inoculated }\end{array}$ & $4.95^{\mathrm{c}}$ & $0.05^{\mathrm{b}}$ & Very less & Not bad & Less sour taste \\
\hline $\begin{array}{l}\mathrm{T}_{7}-\text { Roasted seed, powdered } \\
+ \text { un inoculated }\end{array}$ & $5.26^{\mathrm{d}}$ & $0.07^{\mathrm{bc}}$ & Very less & Good & Less sour taste \\
\hline $\begin{array}{l}\mathrm{T}_{8}-\text { Roasted seed, powdered } \\
+ \text { inoculated }\end{array}$ & $4.73^{\mathrm{b}}$ & $0.13^{\mathrm{bc}}$ & Very less & $\begin{array}{l}\text { Grainy, } \\
\text { good }\end{array}$ & Sour and good \\
\hline
\end{tabular}

Table.3 Sensory characteristics of beverages prepared with varying substrate concentration

\begin{tabular}{|c|c|c|c|c|c|c|c|c|c|c|c|}
\hline \multicolumn{3}{|c|}{ Treatment details } & \multicolumn{9}{|c|}{ Days of incubation } \\
\hline & \multirow{2}{*}{ Organism } & \multirow{2}{*}{$\begin{array}{c}\text { Substrate } \\
\text { concentration }\end{array}$} & \multicolumn{3}{|c|}{ Foam } & \multicolumn{3}{|c|}{ Flavour } & \multicolumn{3}{|c|}{ Taste } \\
\hline & & & 2 & 3 & 4 & 2 & 3 & 4 & 2 & 3 & 4 \\
\hline $\mathbf{T}_{1}$ & \begin{tabular}{|c|}
$L$. \\
acidophilus
\end{tabular} & $5 \%$ & No & No & No & $\begin{array}{l}\text { Good } \\
\text { grainy }\end{array}$ & Good & $\begin{array}{l}\text { Nutty and } \\
\text { grainy }\end{array}$ & $\begin{array}{l}\text { More } \\
\text { sweet }\end{array}$ & Sugary & $\begin{array}{l}\text { Little } \\
\text { sour and } \\
\text { good }\end{array}$ \\
\hline $\mathbf{T}_{2}$ & $\begin{array}{c}L . \\
\text { acidophilus }\end{array}$ & $10 \%$ & No & No & No & Grainy & Good & $\begin{array}{l}\text { Slight } \\
\text { fermented }\end{array}$ & $\begin{array}{l}\text { Sugar } \\
\text { and sour }\end{array}$ & $\begin{array}{l}\text { Sour and } \\
\text { sweet }\end{array}$ & $\begin{array}{l}\text { Sour and } \\
\text { good }\end{array}$ \\
\hline $\mathbf{T}_{3}$ & $\begin{array}{c}\text { L. } \\
\text { acidophilus }\end{array}$ & $15 \%$ & No & No & No & Good & Good & $\begin{array}{l}\text { Grainy } \\
\text { and nutty }\end{array}$ & Sugary & $\begin{array}{l}\text { Sugary } \\
\text { and nutty }\end{array}$ & $\begin{array}{l}\text { Slightly } \\
\text { sour and } \\
\text { sweet }\end{array}$ \\
\hline $\mathbf{T}_{4}$ & $\mathrm{SY}_{2}$ & $5 \%$ & Yes & Yes & Yes & Alcoholic & $\begin{array}{l}\text { Good, } \\
\text { Alcoholic }\end{array}$ & Good & $\begin{array}{l}\text { Not } \\
\text { slimy }\end{array}$ & $\begin{array}{l}\text { Little } \\
\text { sour and } \\
\text { good }\end{array}$ & $\begin{array}{l}\text { Not } \\
\text { good }\end{array}$ \\
\hline $\mathbf{T}_{5}$ & $\mathrm{SY}_{2}$ & $10 \%$ & Yes & Yes & Yes & Alcoholic & Fermented & $\begin{array}{l}\text { Not good } \\
\text { as } T_{2}\end{array}$ & $\begin{array}{l}\text { Little } \\
\text { slimy } \\
\text { and } \\
\text { alcoholic }\end{array}$ & $\begin{array}{l}\text { Gummy } \\
\text { and not } \\
\text { good }\end{array}$ & $\begin{array}{l}\text { Not } \\
\text { good, } \\
\text { gummy }\end{array}$ \\
\hline$T_{6}$ & $\mathrm{SY}_{2}$ & $15 \%$ & Yes & Yes & Yes & $\begin{array}{l}\text { Grainy, } \\
\text { fermented }\end{array}$ & Fermented & $\mathrm{Bad}$ & $\begin{array}{l}\text { More } \\
\text { alcoholic } \\
\text { and } \\
\text { slimy }\end{array}$ & $\begin{array}{l}\text { Too much } \\
\text { slimy and } \\
\text { not good }\end{array}$ & $\begin{array}{l}\text { Not } \\
\text { good, } \\
\text { too } \\
\text { much } \\
\text { gummy }\end{array}$ \\
\hline
\end{tabular}


Table.3A Titrable acidity and $\mathrm{pH}$ of beverages prepared using different substrate concentrations

\begin{tabular}{|c|c|c|c|c|c|c|c|c|}
\hline \multicolumn{3}{|c|}{ Treatment details } & \multicolumn{6}{|c|}{ Days of incubation } \\
\hline \multirow[t]{2}{*}{ Treatment } & \multirow[t]{2}{*}{ Organism } & \multirow{2}{*}{$\begin{array}{c}\text { Substrate } \\
\text { concentration }\end{array}$} & \multicolumn{3}{|c|}{ pH } & \multicolumn{3}{|c|}{ Titrable Acidity } \\
\hline & & & 2 & 3 & 4 & 2 & 3 & 4 \\
\hline $\mathbf{T}_{1}$ & L. acidophilus & $5 \%$ & $4.60^{\mathrm{a}}$ & $4.56^{\mathrm{a}}$ & $4.52^{\mathrm{a}}$ & $0.06^{\mathrm{d}}$ & $0.08^{\mathrm{e}}$ & $0.13^{\mathrm{d}}$ \\
\hline$T_{2}$ & L. acidophilus & $10 \%$ & $4.64^{\mathrm{a}}$ & $4.51^{\mathrm{a}}$ & $4.21^{\mathrm{b}}$ & $0.10^{\mathrm{cd}}$ & $0.15^{\mathrm{e}}$ & $0.29^{\mathrm{c}}$ \\
\hline $\mathbf{T}_{3}$ & L. acidophilus & $15 \%$ & $4.65^{\mathrm{a}}$ & $4.53^{\mathrm{a}}$ & $4.30^{\mathrm{b}}$ & $0.14^{\mathrm{c}}$ & $0.23^{\mathrm{d}}$ & $0.35^{\mathrm{bc}}$ \\
\hline $\mathbf{T}_{4}$ & $\mathbf{S Y}_{2}$ & $5 \%$ & $4.09^{\mathrm{c}}$ & $4.05^{\mathrm{c}}$ & $3.95^{\mathrm{d}}$ & $0.31^{\mathrm{b}}$ & $0.33^{\mathrm{c}}$ & $0.40^{\mathrm{b}}$ \\
\hline $\mathbf{T}_{5}$ & $\mathbf{S Y}_{2}$ & $10 \%$ & $4.09^{\mathrm{c}}$ & $4.08^{\mathrm{bc}}$ & $4.07^{\mathrm{cd}}$ & $0.50^{\mathrm{a}}$ & $0.50^{\mathrm{b}}$ & $0.60^{\mathrm{a}}$ \\
\hline$T_{6}$ & $\mathbf{S Y}_{2}$ & $15 \%$ & $4.19^{\mathrm{b}}$ & $4.16^{\mathrm{b}}$ & $4.15^{\mathrm{bc}}$ & $0.54^{\mathrm{a}}$ & $0.60^{\mathrm{a}}$ & $0.62^{\mathrm{a}}$ \\
\hline
\end{tabular}

Table.4 Sensory characteristics of beverages prepared using different sugar concentrations

\begin{tabular}{|c|c|c|c|c|c|c|c|c|c|c|c|}
\hline \multicolumn{3}{|c|}{ Treatment details } & \multicolumn{9}{|c|}{ Days of incubation } \\
\hline & \multirow[t]{2}{*}{ Organism } & \multirow{2}{*}{$\begin{array}{c}\text { Sugar } \\
\text { concentra } \\
\text { tion }\end{array}$} & \multicolumn{3}{|c|}{ Foam } & \multicolumn{3}{|c|}{ Flavour } & \multicolumn{3}{|c|}{ Taste } \\
\hline & & & 2 & 3 & 4 & 2 & 3 & 4 & 2 & 3 & 4 \\
\hline $\mathbf{T}_{1}$ & L. acidophilus & $5 \%$ & No & No & No & $\begin{array}{l}\text { Slightly } \\
\text { grain }\end{array}$ & Good & Grainy & $\begin{array}{l}\text { More } \\
\text { sweet } \\
\text { and } \\
\text { slightly } \\
\text { sour }\end{array}$ & $\begin{array}{l}\text { Sour } \\
\text { taste }\end{array}$ & $\begin{array}{l}\text { Slightly } \\
\text { sour }\end{array}$ \\
\hline $\mathbf{T}_{2}$ & L. acidophilus & $6.6 \%$ & No & No & No & More grainy & Sour & Sour & $\begin{array}{l}\text { Little } \\
\text { sweet } \\
\text { and sour }\end{array}$ & $\begin{array}{l}\text { Sour } \\
\text { taste }\end{array}$ & $\begin{array}{l}\text { Slight } \\
\text { sweet } \\
\text { and } \\
\text { more } \\
\text { sour }\end{array}$ \\
\hline $\mathbf{T}_{3}$ & L. acidophilus & $8 \%$ & No & No & No & $\begin{array}{l}\text { grainy and } \\
\text { light sour }\end{array}$ & $\begin{array}{l}\text { Sour and } \\
\text { grainy }\end{array}$ & Sour & $\begin{array}{l}\text { sweet } \\
\text { and little } \\
\text { sour }\end{array}$ & $\begin{array}{l}\text { Sour } \\
\text { and } \\
\text { sweet }\end{array}$ & Sour \\
\hline $\mathbf{T}_{4}$ & L. acidophilus & $10 \%$ & No & No & No & Grainy & Fermented & $\begin{array}{l}\text { Slight } \\
\text { fermented }\end{array}$ & $\begin{array}{l}\text { Sugar } \\
\text { and sour }\end{array}$ & Bitter & $\begin{array}{l}\text { Sour } \\
\text { and } \\
\text { good }\end{array}$ \\
\hline $\mathbf{T}_{5}$ & $\mathrm{SY}_{2}$ & $5 \%$ & Yes & Yes & Yes & Fermented & Off flavour & Off flavour & $\begin{array}{l}\text { Sour } \\
\text { and } \\
\text { sweet }\end{array}$ & Bitter & Bitter \\
\hline$T_{6}$ & $\mathbf{S Y}_{2}$ & $6.6 \%$ & Yes & Yes & Yes & $\begin{array}{l}\text { Little off- } \\
\text { flavour }\end{array}$ & Off flavour & Off flavour & bitter & Bitter & Bitter \\
\hline $\mathbf{T}_{7}$ & $\mathbf{S Y}_{2}$ & $8 \%$ & Yes & Yes & Yes & $\begin{array}{l}\text { Little off- } \\
\text { flavour }\end{array}$ & Off flavour & Off flavour & bitter & $\begin{array}{l}\text { Little } \\
\text { sour } \\
\text { and } \\
\text { good } \\
\end{array}$ & Bitter \\
\hline $\mathbf{T}_{8}$ & $\mathrm{SY}_{2}$ & $10 \%$ & Yes & Yes & Yes & Alcoholic & Alcoholic & Good & $\begin{array}{l}\text { Not } \\
\text { slimy }\end{array}$ & $\begin{array}{l}\text { Sweet } \\
\text { and } \\
\text { little } \\
\text { sour }\end{array}$ & good \\
\hline
\end{tabular}


Table.4A Titrable acidity and $\mathrm{pH}$ of beverages prepared with varying sugar concentrations

\begin{tabular}{|c|c|c|c|c|c|c|c|c|}
\hline \multicolumn{3}{|c|}{ Treatment details } & \multicolumn{6}{|c|}{ Days of incubation } \\
\hline \multirow[t]{2}{*}{ Treatment } & \multirow[t]{2}{*}{ Organism } & \multirow{2}{*}{$\begin{array}{c}\text { Sugar } \\
\text { concentration }\end{array}$} & \multicolumn{3}{|c|}{ pH } & \multicolumn{3}{|c|}{ Titrable Acidity } \\
\hline & & & 2 & 3 & 4 & 2 & 3 & 4 \\
\hline $\mathbf{T}_{1}$ & L. acidophilus & $5 \%$ & $4.34^{\mathrm{ab}}$ & $4.11^{f}$ & $4.01^{\mathrm{b}}$ & $0.13^{\mathrm{d}}$ & $0.22^{\mathrm{bc}}$ & $0.27^{\mathrm{b}}$ \\
\hline $\mathbf{T}_{2}$ & L. acidophilus & $6.6 \%$ & $4.36^{\mathrm{bc}}$ & $4.13^{\mathrm{e}}$ & $3.99^{b}$ & $0.14^{\mathrm{cd}}$ & $0.23^{\mathrm{bc}}$ & $0.27^{\mathrm{b}}$ \\
\hline $\mathbf{T}_{3}$ & L. acidophilus & $8 \%$ & $4.41^{\mathrm{c}}$ & $4.10^{\mathrm{f}}$ & $3.98^{\mathrm{b}}$ & $0.11^{\mathrm{cd}}$ & $0.22^{\mathrm{c}}$ & $0.24^{\mathrm{b}}$ \\
\hline $\mathbf{T}_{4}$ & L. acidophilus & $10 \%$ & $4.64^{\mathrm{a}}$ & $4.51^{\mathrm{a}}$ & $4.21^{\mathrm{a}}$ & $0.10^{\mathrm{bc}}$ & $0.15^{\mathrm{d}}$ & $0.29^{b}$ \\
\hline $\mathbf{T}_{5}$ & $\mathrm{SY}_{2}$ & $5 \%$ & $4.50^{\mathrm{a}}$ & $4.23^{\mathrm{b}}$ & $3.98^{b}$ & $0.28^{\mathrm{a}}$ & $0.29^{\mathrm{a}}$ & $0.31^{\mathrm{b}}$ \\
\hline$T_{6}$ & $\mathrm{SY}_{2}$ & $6.6 \%$ & $4.46^{\mathrm{a}}$ & $4.22^{\mathrm{c}}$ & $4.00^{\mathrm{b}}$ & $0.26^{\mathrm{ab}}$ & $0.28^{\mathrm{ab}}$ & $0.28^{\mathrm{b}}$ \\
\hline $\mathbf{T}_{7}$ & $\mathrm{SY}_{2}$ & $8 \%$ & $4.43^{\mathrm{a}}$ & $4.19^{\mathrm{d}}$ & $3.99^{b}$ & $0.24^{\mathrm{ab}}$ & $0.28^{\mathrm{ab}}$ & $0.29^{b}$ \\
\hline $\mathbf{T}_{8}$ & $\mathrm{SY}_{2}$ & $10 \%$ & $4.09^{c}$ & $4.05^{\mathrm{g}}$ & $3.95^{\mathrm{b}}$ & $0.31^{\mathrm{a}}$ & $0.33^{\mathrm{a}}$ & $0.40^{\mathrm{a}}$ \\
\hline
\end{tabular}

Table.5 Sensory characteristics of beverages prepared using different inoculum concentrations

\begin{tabular}{|c|c|c|c|c|c|c|c|c|c|c|c|}
\hline \multicolumn{3}{|c|}{ Treatment details } & \multicolumn{9}{|c|}{ Days of incubation } \\
\hline & \multirow[t]{2}{*}{ Organism } & \multirow{2}{*}{$\begin{array}{l}\text { Inoculum } \\
\text { concentrati } \\
\text { on }\end{array}$} & \multicolumn{3}{|c|}{ Foam } & \multicolumn{3}{|c|}{ Flavour } & \multicolumn{3}{|c|}{ Taste } \\
\hline & & & 2 & 3 & 4 & 2 & 3 & 4 & 2 & 3 & 4 \\
\hline $\mathbf{T}_{1}$ & L. acidophilus & $6 \%$ & No & No & No & Grainy & $\begin{array}{l}\text { Good } \\
\text { grainy }\end{array}$ & $\begin{array}{l}\text { Grainy and } \\
\text { a little sour }\end{array}$ & Sweet & $\begin{array}{l}\text { Sour and } \\
\text { sweet }\end{array}$ & $\begin{array}{l}\text { Sour } \\
\text { and } \\
\text { sweet }\end{array}$ \\
\hline $\mathbf{T}_{2}$ & L. acidophilus & $8 \%$ & No & No & No & Good & Good & $\begin{array}{l}\text { Sour and } \\
\text { Grainy }\end{array}$ & $\begin{array}{l}\text { Sour than } \\
\mathrm{T}_{1}\end{array}$ & $\begin{array}{l}\text { Sour as } \\
\text { same as } \\
\mathrm{T}_{3}\end{array}$ & $\begin{array}{l}\text { More } \\
\text { sour }\end{array}$ \\
\hline $\mathbf{T}_{3}$ & L. acidophilus & $10 \%$ & No & No & No & Grainy & Little sour & Sour & $\begin{array}{l}\text { Sour than } \\
\mathrm{T}_{1}\end{array}$ & $\begin{array}{l}\text { Sour and } \\
\text { good }\end{array}$ & $\begin{array}{l}\text { More } \\
\text { sour }\end{array}$ \\
\hline $\mathbf{T}_{4}$ & $\mathbf{S Y}_{2}$ & $6 \%$ & Yes & Yes & Yes & fermented & fermented & $\begin{array}{l}\text { More } \\
\text { fermented }\end{array}$ & $\begin{array}{l}\text { Sweet and } \\
\text { a little } \\
\text { bitter }\end{array}$ & $\begin{array}{l}\text { Little } \\
\text { bitter }\end{array}$ & Bitter \\
\hline $\mathbf{T}_{5}$ & $\overline{\mathbf{S Y}_{2}}$ & $8 \%$ & Yes & Yes & yes & Fermented & Fermented & $\begin{array}{l}\text { More } \\
\text { fermented }\end{array}$ & $\begin{array}{l}\text { Sour and } \\
\text { good }\end{array}$ & $\begin{array}{l}\text { Sour and } \\
\text { good }\end{array}$ & Bitter \\
\hline$T_{6}$ & $\mathbf{S Y}_{2}$ & $10 \%$ & Yes & yes & Yes & Fermented & $\begin{array}{l}\text { More } \\
\text { fermented }\end{array}$ & $\begin{array}{l}\text { Little off- } \\
\text { flavour }\end{array}$ & $\begin{array}{l}\text { Sour and } \\
\text { bitter }\end{array}$ & $\begin{array}{l}\text { Sour and } \\
\text { bitter }\end{array}$ & Bitter \\
\hline
\end{tabular}

Table.5A Titrable acidity and $\mathrm{pH}$ of beverages prepared using different inoculum concentrations

\begin{tabular}{|c|c|c|c|c|c|c|c|c|}
\hline \multicolumn{3}{|c|}{ Treatment details } & \multicolumn{5}{|c|}{ Days of incubation } \\
\hline Treatment & Organism & \multirow{2}{*}{$\begin{array}{c}\text { Inoculum } \\
\text { concentration }\end{array}$} & & \multicolumn{3}{|c|}{$\mathbf{p H}$} & \multicolumn{3}{|c|}{ Titrable Acidity } \\
\cline { 4 - 9 } & & $\mathbf{6}$ & $\mathbf{3}$ & $\mathbf{4}$ & $\mathbf{2}$ & $\mathbf{3}$ & $\mathbf{4}$ \\
\hline $\mathbf{T}_{\mathbf{1}}$ & L. acidophilus & $\mathbf{6} \%$ & $4.62^{\mathrm{a}}$ & 4.03 & $3.97^{\mathrm{a}}$ & $0.15^{\mathrm{b}}$ & $0.22^{\mathrm{c}}$ & $0.27^{\mathrm{d}}$ \\
\hline $\mathbf{T}_{\mathbf{2}}$ & L. acidophilus & $\mathbf{8} \%$ & $4.43^{\mathrm{b}}$ & 4.08 & $3.97^{\mathrm{a}}$ & $0.14^{\mathrm{b}}$ & $0.21^{\mathrm{c}}$ & $0.26^{\mathrm{d}}$ \\
\hline $\mathbf{T}_{\mathbf{3}}$ & L. acidophilus & $\mathbf{1 0} \%$ & $4.25^{\mathrm{d}}$ & 4.00 & $3.95^{\mathrm{a}}$ & $0.15^{\mathrm{b}}$ & $0.20^{\mathrm{c}}$ & $0.29^{\mathrm{cd}}$ \\
\hline $\mathbf{T}_{\mathbf{4}}$ & $\mathbf{S Y}_{\mathbf{2}}$ & $\mathbf{6 \%}$ & $4.38^{\mathrm{bc}}$ & 4.01 & $3.73^{\mathrm{b}}$ & $0.23^{\mathrm{a}}$ & $0.29^{\mathrm{b}}$ & $0.37^{\mathrm{bc}}$ \\
\hline $\mathbf{T}_{\mathbf{5}}$ & $\mathbf{S Y}_{\mathbf{2}}$ & $\mathbf{8 \%}$ & $4.34^{\mathrm{c}}$ & 3.99 & $3.70^{\mathrm{b}}$ & $0.23^{\mathrm{a}}$ & $0.32^{\mathrm{ab}}$ & $0.46^{\mathrm{a}}$ \\
\hline $\mathbf{T}_{\mathbf{6}}$ & $\mathbf{S Y}_{\mathbf{2}}$ & $\mathbf{1 0} \%$ & $4.30^{\mathrm{cd}}$ & 3.93 & $3.71^{\mathrm{b}}$ & $0.23^{\mathrm{a}}$ & $0.35^{\mathrm{a}}$ & $0.44^{\mathrm{ab}}$ \\
\hline
\end{tabular}


Table.6 Sensory characteristics of beverages prepared using different prebiotic (honey) concentrations

\begin{tabular}{|c|c|c|c|c|c|c|c|c|}
\hline \multicolumn{3}{|c|}{ Treatment details } & \multicolumn{6}{|c|}{ Days of incubation } \\
\hline & \multirow{2}{*}{ Inoculum } & \multirow{2}{*}{$\begin{array}{l}\text { Sugar: } \\
\text { Honey }\end{array}$} & \multicolumn{2}{|c|}{ Foam } & \multicolumn{2}{|c|}{ Flavour } & \multicolumn{2}{|c|}{ Taste } \\
\hline & & & 2 & 3 & 2 & 3 & 2 & 3 \\
\hline $\mathbf{T}_{1}$ & L. acidophilus & 100:0 & No & No & Sweet & $\begin{array}{c}\text { Sweet and } \\
\text { grainy }\end{array}$ & More sweet & Little sweet \\
\hline $\mathbf{T}_{2}$ & L. acidophilus & 80:20 & No & No & Good, grainy & $\begin{array}{l}\text { Good, } \\
\text { grainy }\end{array}$ & $\begin{array}{l}\text { Sour and } \\
\text { good }\end{array}$ & $\begin{array}{l}\text { Sour and a } \\
\text { little sweet }\end{array}$ \\
\hline $\mathbf{T}_{3}$ & L. acidophilus & $60: 40$ & No & No & Grainy, good & Good & $\begin{array}{l}\begin{array}{l}\text { Sweet and } \\
\text { sour (not } \\
\text { sour than } \mathrm{T}_{2} \\
\text { and } \mathrm{T}_{4} \text { ) }\end{array} \\
\end{array}$ & More sour \\
\hline $\mathbf{T}_{4}$ & L. acidophilus & $50: 50$ & No & No & Good, grainy & $\begin{array}{c}\text { Little } \\
\text { fermented }\end{array}$ & More sour & More sour \\
\hline $\mathbf{T}_{5}$ & $\mathrm{SY}_{2}$ & $100: 0$ & Yes & Yes & $\begin{array}{c}\text { Good, } \\
\text { fermented }\end{array}$ & Good & $\begin{array}{l}\text { Sweet and } \\
\text { good }\end{array}$ & Little bitter \\
\hline $\mathbf{T}_{6}$ & $\mathrm{SY}_{2}$ & $35: 65$ & Yes & Yes & $\begin{array}{c}\text { Grainy, } \\
\text { fermented }\end{array}$ & $\begin{array}{c}\text { Good, } \\
\text { fermented }\end{array}$ & Sweet & $\begin{array}{l}\text { Sour and } \\
\text { good }\end{array}$ \\
\hline $\mathbf{T}_{7}$ & $\mathbf{S Y}_{2}$ & $50: 50$ & Yes & Yes & $\begin{array}{l}\text { Good (better } \\
\text { than } \mathrm{T}_{8} \text { ) }\end{array}$ & Grainy & $\begin{array}{l}\text { Sour and } \\
\text { good }\end{array}$ & $\begin{array}{c}\text { Sour and } \\
\text { good }\end{array}$ \\
\hline $\mathbf{T}_{8}$ & $\mathbf{S Y}_{2}$ & $65: 35$ & Yes & Yes & $\begin{array}{c}\text { Grainy, } \\
\text { fermented }\end{array}$ & Fermented & $\begin{array}{l}\text { Sour and a } \\
\text { little sweet }\end{array}$ & More sour \\
\hline
\end{tabular}

Table.6A Titrable acidity and $\mathrm{pH}$ of beverages prepared using different prebiotic (honey) concentrations

\begin{tabular}{|c|c|c|c|c|c|c|}
\hline \multicolumn{3}{|c|}{ Treatment details } & \multicolumn{4}{|c|}{ Days of incubation } \\
\hline \multirow[t]{2}{*}{ Treatment } & \multirow[t]{2}{*}{ Inoculum } & \multirow[t]{2}{*}{ Sugar: Honey } & \multicolumn{2}{|c|}{ pH } & \multicolumn{2}{|c|}{ Titrable Acidity } \\
\hline & & & 2 & 3 & 2 & 3 \\
\hline $\mathbf{T}_{1}$ & L. acidophilus & $100: 0$ & $4.52^{\mathrm{a}}$ & $4.08^{\mathrm{a}}$ & $0.13^{\mathrm{e}}$ & $0.41^{\mathrm{e}}$ \\
\hline $\mathbf{T}_{2}$ & L. acidophilus & $80: 20$ & $4.30^{\mathrm{cd}}$ & $3.98^{\mathrm{a}}$ & $0.28^{\mathrm{b}}$ & $0.49^{\mathrm{de}}$ \\
\hline $\mathbf{T}_{3}$ & L. acidophilus & $60: 40$ & $4.20^{\mathrm{bc}}$ & $3.91^{\mathrm{b}}$ & $0.20^{\mathrm{d}}$ & $0.71^{\mathrm{bcc}}$ \\
\hline $\mathbf{T}_{4}$ & L. acidophilus & $50: 50$ & $4.20^{\mathrm{bc}}$ & $3.71^{\mathrm{c}}$ & $0.23^{\text {cd }}$ & $0.73^{\mathrm{bcc}}$ \\
\hline $\mathbf{T}_{5}$ & $\mathrm{SY}_{2}$ & 100:0 & $4.27^{\mathrm{b}}$ & $3.66^{\mathrm{c}}$ & $0.26^{\mathrm{bc}}$ & $0.55^{\mathrm{cdc}}$ \\
\hline$T_{6}$ & $\mathrm{SY}_{2}$ & $35: 65$ & $4.20^{\mathrm{bc}}$ & $3.62^{\mathrm{c}}$ & $0.38^{\mathrm{a}}$ & $0.74^{\mathrm{bc}}$ \\
\hline $\mathbf{T}_{7}$ & $\mathrm{SY}_{2}$ & $50: 50$ & $3.99^{\mathrm{d}}$ & $3.47^{\mathrm{d}}$ & $0.28^{\mathrm{b}}$ & $0.88^{\mathrm{ab}}$ \\
\hline $\mathbf{T}_{8}$ & $\mathrm{SY}_{2}$ & $65: 35$ & $4.20^{\mathrm{bc}}$ & $3.50^{\mathrm{d}}$ & $0.29^{\mathrm{b}}$ & $1.11^{\mathrm{a}}$ \\
\hline
\end{tabular}


Table.7 Sensory characteristics of beverages incubated at different temperatures

\begin{tabular}{|c|c|c|c|c|c|c|c|c|}
\hline \multicolumn{3}{|c|}{ Treatment details } & \multicolumn{6}{|c|}{ Days of incubation } \\
\hline & \multirow[t]{2}{*}{ Inoculum } & \multirow{2}{*}{$\begin{array}{l}\text { Incubation } \\
\text { temperature }\end{array}$} & \multicolumn{2}{|c|}{ Foam } & \multicolumn{2}{|c|}{ Flavour } & \multicolumn{2}{|c|}{ Taste } \\
\hline & & & 2 & 3 & 2 & 3 & 2 & 3 \\
\hline $\mathbf{T}_{1}$ & L. acidophilus & $28^{\circ} \mathrm{C}$ & No & No & $\begin{array}{l}\text { Good } \\
\text { grainy } \\
\text { smell }\end{array}$ & $\begin{array}{l}\text { Grainy } \\
\text { and } \\
\text { fermented } \\
\text { smell }\end{array}$ & Sour & $\begin{array}{l}\text { More } \\
\text { sour }\end{array}$ \\
\hline $\mathbf{T}_{2}$ & L. acidophilus & $30^{\circ} \mathrm{C}$ & No & No & $\begin{array}{l}\text { Grainy and } \\
\text { fermented } \\
\text { smell }\end{array}$ & $\begin{array}{l}\text { fermented } \\
\text { smell, } \\
\text { good }\end{array}$ & $\begin{array}{l}\text { Sweet, a } \\
\text { little } \\
\text { sour }\end{array}$ & Sour \\
\hline $\mathbf{T}_{3}$ & L. acidophilus & $32^{\circ} \mathrm{C}$ & No & No & $\begin{array}{l}\text { Grainy and } \\
\text { fermented } \\
\text { smell }\end{array}$ & $\begin{array}{l}\text { fermented } \\
\text { smell, } \\
\text { good }\end{array}$ & $\begin{array}{l}\text { Sweet } \\
\text { and sour }\end{array}$ & Sour \\
\hline $\mathbf{T}_{4}$ & $\mathrm{SY}_{2}$ & $28^{\circ} \mathrm{C}$ & Yes & Yes & $\begin{array}{l}\text { Fermented } \\
\text { smell }\end{array}$ & $\begin{array}{l}\text { Fermented } \\
\text { smell }\end{array}$ & $\begin{array}{l}\text { More } \\
\text { sour and } \\
\text { good }\end{array}$ & $\begin{array}{l}\text { Sour } \\
\text { and a } \\
\text { little } \\
\text { bitter }\end{array}$ \\
\hline $\mathbf{T}_{5}$ & $\mathbf{S Y}_{2}$ & $3^{\circ}{ }^{\circ} \mathrm{C}$ & Yes & Yes & $\begin{array}{l}\text { Little } \\
\text { fermented } \\
\text { smell }\end{array}$ & $\begin{array}{l}\text { Little } \\
\text { fermented } \\
\text { smell }\end{array}$ & $\begin{array}{l}\text { Sweet } \\
\text { and } \\
\text { good }\end{array}$ & $\begin{array}{l}\text { Sour } \\
\text { and } \\
\text { good }\end{array}$ \\
\hline$T_{6}$ & $\mathbf{S Y}_{2}$ & $32^{\circ} \mathrm{C}$ & Yes & Yes & $\begin{array}{l}\text { Fermented } \\
\text { smell }\end{array}$ & $\begin{array}{l}\text { fermented } \\
\text { smell }\end{array}$ & $\begin{array}{l}\text { Sour } \\
\text { and } \\
\text { sweet }\end{array}$ & $\begin{array}{l}\text { Sour } \\
\text { and } \\
\text { good }\end{array}$ \\
\hline
\end{tabular}

Table.7A Titrable acidity and $\mathrm{pH}$ of beverages incubated at different temperatures

\begin{tabular}{|c|c|c|c|c|c|c|}
\hline \multicolumn{3}{|c|}{ Treatment details } & \multicolumn{4}{|c|}{ Days of incubation } \\
\hline \multirow[t]{2}{*}{ Treatment } & \multirow[t]{2}{*}{ Organism } & \multirow{2}{*}{$\begin{array}{l}\text { Incubation } \\
\text { temperature }\end{array}$} & \multicolumn{2}{|c|}{ pH } & \multicolumn{2}{|c|}{ Titrable Acidity } \\
\hline & & & 2 & 3 & 2 & 3 \\
\hline$T_{1}$ & L.acidophilus & $28^{\circ} \mathrm{C}$ & $4.04^{\mathrm{b}}$ & $3.98^{\mathrm{cd}}$ & $0.34^{\mathrm{a}}$ & $0.55^{\mathrm{a}}$ \\
\hline $\mathbf{T}_{2}$ & L.acidophilus & $30^{\circ} \mathrm{C}$ & $4.20^{\mathrm{a}}$ & $4.10^{\mathrm{b}}$ & $0.10^{\mathrm{d}}$ & $0.51^{\mathrm{b}}$ \\
\hline $\mathbf{T}_{3}$ & L.acidophilus & $32^{\circ} \mathrm{C}$ & $4.11^{\mathrm{ab}}$ & $4.13^{\mathrm{a}}$ & $0.14^{\mathrm{cd}}$ & $0.26^{\mathrm{e}}$ \\
\hline $\mathbf{T}_{4}$ & $\mathrm{SY}_{2}$ & $28^{\circ} \mathrm{C}$ & $4.03^{\mathrm{b}}$ & $3.92^{\mathrm{d}}$ & $0.19^{\mathrm{bc}}$ & $0.38^{\mathrm{c}}$ \\
\hline $\mathbf{T}_{5}$ & $\mathrm{SY}_{2}$ & $30^{\circ} \mathrm{C}$ & $4.15^{\mathrm{a}}$ & $4.02^{\mathrm{bc}}$ & $0.17^{\mathrm{bc}}$ & $0.34^{\mathrm{d}}$ \\
\hline$T_{6}$ & $\mathrm{SY}_{2}$ & $32^{\circ} \mathrm{C}$ & $3.93^{\mathrm{c}}$ & $3.82^{\mathrm{e}}$ & $0.24^{\mathrm{b}}$ & $0.27^{\mathrm{e}}$ \\
\hline
\end{tabular}


Table.8 Standardization of optimum fermentation duration for the preparation of linseed beverage

\begin{tabular}{|c|c|c|c|c|c|c|c|}
\hline \multicolumn{3}{|c|}{ Treatment details } & \multirow{2}{*}{ PH } & \multirow{2}{*}{$\begin{array}{c}\text { Titrable } \\
\text { acidity }\end{array}$} & Foam & Flavour & Taste \\
\cline { 1 - 6 } & Inoculum & $\begin{array}{c}\text { Duration } \\
\text { (Days) }\end{array}$ & & & & & \\
\hline $\mathbf{T}_{\mathbf{1}}$ & L. acidophilus & $\mathbf{2}$ & $4.43^{\mathrm{a}}$ & $0.18^{\mathrm{d}}$ & No & $\begin{array}{c}\text { Grainy and } \\
\text { fermented }\end{array}$ & Sour and little sweet \\
\hline $\mathbf{T}_{\mathbf{2}}$ & $\mathbf{S Y}_{\mathbf{2}}$ & $\mathbf{2}$ & $4.32^{\mathrm{b}}$ & $0.19^{\mathrm{d}}$ & Yes & Fermented & Little sweet and sour \\
\hline $\mathbf{T}_{\mathbf{3}}$ & L. acidophilus & $\mathbf{3}$ & $3.90^{\mathrm{c}}$ & $0.25^{\mathrm{c}}$ & No & Fermented, good & Sour and good \\
\hline $\mathbf{T}_{\mathbf{4}}$ & $\mathbf{S Y}_{\mathbf{2}}$ & $\mathbf{3}$ & $3.92^{\mathrm{c}}$ & $0.24^{\mathrm{c}}$ & Yes & Fermented & Sour and good \\
\hline $\mathbf{T}_{\mathbf{5}}$ & L. acidophilus & $\mathbf{4}$ & $3.76^{\mathrm{d}}$ & $0.54^{\mathrm{a}}$ & No & More fermented & More sour \\
\hline $\mathbf{T}_{\mathbf{6}}$ & $\mathbf{S Y}_{\mathbf{2}}$ & $\mathbf{4}$ & $3.68^{\mathrm{e}}$ & $0.43^{\mathrm{b}}$ & Yes & More fermented & More sour and good \\
\hline
\end{tabular}

Table.9 Mean scores of organoleptic evaluation of the fermented linseed beverage prepared using lactic acid bacteria and yeasts

\begin{tabular}{|l|c|c|c|c|c|c|c|c|}
\hline Treatments & Appearance & Colour & Texture & Taste & Aroma & Astringent & $\begin{array}{c}\text { Mouth } \\
\text { feel }\end{array}$ & $\begin{array}{c}\text { overall } \\
\text { acceptability }\end{array}$ \\
\hline $\mathbf{T}_{\mathbf{1}}$ (Lactobacillus & $7.1 \pm 0.70$ & $7.0 \pm$ & $7.3 \pm$ & $7.5 \pm$ & $7.8 \pm$ & $6.8 \pm 1.19$ & $6.9 \pm$ & $7.5 \pm 0.87$ \\
acidophilus) & & 0.71 & 1.05 & 1.12 & 0.66 & & 1.54 & \\
\hline $\mathbf{T}_{\mathbf{2}}$ (Bacillus & $7.1 \pm 0.78$ & $7.1 \pm$ & $7.1 \pm$ & $7.1 \pm$ & $6.8 \pm$ & $6.6 \pm 0.78$ & $6.9 \pm$ & $6.8 \pm 0.95$ \\
mesentericus) & & 0.83 & 0.90 & 0.66 & 1.19 & & 1.05 & \\
\hline $\mathbf{T}_{\mathbf{3}}$ (Lactic acid & $6.9 \pm 0.99$ & $6.8 \pm$ & $6.9 \pm$ & $6.8 \pm$ & $6.8 \pm$ & $6.5 \pm 1.18$ & $7.0 \pm$ & $7.0 \pm 1.12$ \\
bacterial isolate) & & 1.20 & 1.41 & 1.42 & 1.47 & & 1.22 & \\
\hline $\mathbf{T}_{\mathbf{4}}$ (Saccharomyces & $6.9 \pm 1.22$ & $7.1 \pm$ & $6.6 \pm$ & $6.5 \pm$ & $6.5 \pm$ & $6.9 \pm 1.36$ & $6.6 \pm$ & $6.8 \pm 1.20$ \\
ellipsoideus) & & 1.32 & 1.33 & 0.94 & 1.62 & & 1.37 & \\
\hline $\mathbf{T}_{\mathbf{5}}$ (Saccharomyces \\
boulardii)
\end{tabular}

Table.10 Amount of recovery and cost of linseed fermented beverage using probiotic yeasts and lactic acid bacteria

\begin{tabular}{|l|l|c|c|}
\hline \multicolumn{2}{|c|}{ Treatment details } & $\begin{array}{c}\text { Amount of beverage } \\
\text { recovered / 1000 } \mathbf{~ m l} \\
(\mathbf{m l})\end{array}$ & $\begin{array}{c}\text { Cost of production of } \\
\text { beverage/ 1000 } \mathbf{~ m} \\
\text { (rupees) }\end{array}$ \\
\cline { 1 - 2 } Treatment & Inoculum & 588.89 & 51.45 \\
\hline $\mathbf{T}_{\mathbf{1}}$ & Lactobacillus acidophilus & 564.45 & 53.67 \\
\hline $\mathbf{T}_{\mathbf{2}}$ & Bacillus mesentericus & 415.54 & 72.91 \\
\hline $\mathbf{T}_{\mathbf{3}}$ & Isolate LAB-3 & 684.45 & 34.00 \\
\hline $\mathbf{T}_{\mathbf{4}}$ & Saccharomyces ellipsoideus & 704.44 & 33.04 \\
\hline $\mathbf{T}_{\mathbf{5}}$ & Saccharomyces boulardii & & \\
\hline
\end{tabular}

Note - cost of ingredients linseed (1000 g) - Rs. 200, Sugar (1000 g) - Rs. 40, Honey (1000 ml) - Rs. 270, Inoculum $(100 \mathrm{ml}=10 \mathrm{~g}$ linseed $+3 \mathrm{~g}$ sugar $)$ - Rs. 2.12 
Fig.1 Standardized protocol for the preparation of fermented linseed beverage

\section{Preparation of fermented linseed beverage}

Mother culture - Mass production of Lactic acid bacteria and yeasts inocula was done using MRS and yeast extract peptone dextrose broth and incubated at room temperature.

Starter culture - Roasted linseed powder (10\%), Sugar $(3 \%)$ and water was mixed, heated at $60^{\circ} \mathrm{C}$ for 20 min, cooled, filtered using muslin cloth and autoclaved at $121^{\circ} \mathrm{C}, 15 \mathrm{psi}$ for $20 \mathrm{~min}$. Starter medium was inoculated with $10 \%$ mother culture and incubated at room temperature for 36 to $48 \mathrm{hrs}$ and stored at $4{ }^{\circ} \mathrm{C}$.

Seed processing - Linseed was roasted at $60^{\circ} \mathrm{C}$ for $10-15 \mathrm{~min}$, cooled and powdered using mixer.

\section{Beverage preparation}

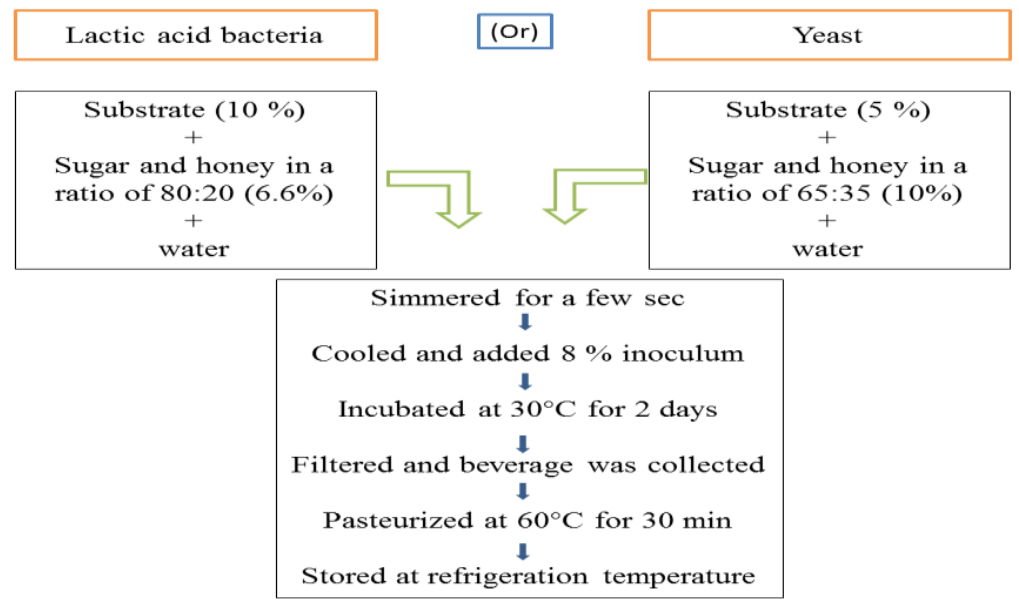

Incubation temperature $28^{\circ} \mathrm{C}, 30^{\circ} \mathrm{C}$ and $32^{\circ} \mathrm{C}$ were tested for fermentation (Table 7 and 7A). Incubation at $32^{\circ} \mathrm{C}$ yielded significant reduction in $\mathrm{pH}$ of 4.11 and 3.93 , more titrable acid production 0.142 per cent and 0.239 per cent and favorable sensory characters for both bacteria and yeast fermentations respectively. Favorable $\mathrm{pH}$ (4.43 and 4.32), Titrable acidity $(0.178 \%$ and $0.192 \%)$ and sensory characteristics including good and fermented flavour with sour and little sweet taste was obtained on $2^{\text {nd }}$ day of lactic acid bacterial and yeast fermentation. So incubation at $32^{\circ} \mathrm{C}$ for 2 days was selected for beverage preparation (Table 8).

Fermentation reported to reduce the $\mathrm{pH}$ and increase the titrable acidity of the sample. During standardization studies, some fermented linseed beverages with same $\mathrm{pH}$ reported to contain different amount of titrable acid. Titrable acidity is a simple measure of the related amount of acid anions in the sample and $\mathrm{pH}$ is a negative logarithm of concentration of free hydrogen ions. There is no direct relationship between titrable acidity and $\mathrm{pH}$, although generally the $\mathrm{pH}$ decreases as the titrable acidity increases and vice-versa. The exact relationship differs from sample to sample and depends on various factors like buffering capacity of acid produced (Jolicoeur, 2011).

Foaming was observed in linseed beverages fermented using yeast and this could be due to the capacity of yeasts in the conversion of sugars into acid, alcohol and carbon dioxide. Absence of foam in lactic acid fermented beverage is due to the homo-fermentative nature of Lactobacillus acidophilus used in the investigation. The experiments were conducted 
at room temperature before standardizing incubation temperature. Variation in biochemical and sensory characteristics of fermented beverages with same ingredients during standardization studies may be due to the differences in the activity and carbohydrate utilization ability of yeasts and lactic acid bacteria at varying room temperature.

Standardized protocol for the preparation of linseed beverage is explained in figure 1 . Fermented beverages were prepared using probiotic organisms. Sensory evaluation (Table 9) recorded a mean score of 6.0 out of 9.0 (liked slightly) to 7.0 out of 9.0 (liked moderately). Beverage recovery after fermentation was studied.

Beverage recovery (Table 10) was more in beverages fermented using yeasts compared to that of lactic acid bacteria and cost of the beverage prepared using lactic acid bacteria was more. Amount of water retained is directly related to the substrate concentration and lactic acid bacterial fermentation involves more amount of substrate than yeasts fermentation. So amount of beverage recovery is less and cost of the beverage is more in lactic acid bacterial fermentation.

In conclusion the growing demand for food with nutritional and sensory quality as well as functional significance calls upon research to develop new products with consumer acceptance. A nutritionally rich beverage from linseed by fermentation was prepared as value addition. The developed protocol can be used for large scale production and further industrialization.

\section{Acknowledgement}

Authors gratefully acknowledge the Department of Agricultural Microbiology, University of Agricultural Sciences, GKVK, Bengaluru - 65 for the financial assistance and laboratory facilities for the completion of work.

\section{References}

Giada, M.L.R. 2010. Food applications for flaxseed and its components: products and processing, Recent Patents Food Nutr. Agric., 2: 181-186.

Jolicoeur, C. 2011. Acidity and pH of apple juice.

http://cjoliprsf.ca/Documents/AciditypH.pdf

Macedoi, L.N., Luchesei, R.H., Guerrai, A.F. and Barbosaii, C.G. 2008. Honey prebiotic effect on the growth and viability of Bifidobacterium spp. and Lactobacillus spp. in milk. Food Sci. Technol., 28: 935-942.

Morris, D.H. 2003. Flax - a health and nutrition primer, IV Edition, Available from: www.flaxcouncil.ca

Panesar, S.P., Kennedy, J.F., Charles, J.K. and Maria, K. 2010. Production of L(+) lactic acid using Lactobacillus casei from whey. Braz. Arch. Boil. Technol., 53: 219-226.

Salminen, K., Kauppinen, V., Virtanen, T., Rajaniemi, S. and Ryhänen, E. 2008. Fermented food product. Patent Ep, 986.

Srivastava, R.P. and Kumar, S. 1993. Important methods for analysis of fruits/vegetable and their products. Fruit Veg. Pres. Prin. Prac., 2: 321-229.

\section{How to cite this article:}

Nivetha, N., V.C. Suvarna and Shraddha, A.J. 2017. Standardization of Protocol for the Preparation of Linseed Beverage (Fermented) Using Lactic Acid Bacteria and Yeast. Int.J.Curr.Microbiol.App.Sci. 6(3): 1628-1638. doi: https://doi.org/10.20546/ijcmas.2017.603.188 